\title{
Laboreal
}

Volume $6 \mathrm{~N}^{\circ} 1$ | 2010

Varia

\section{La violencia laboral hacia trabajadores/as de instituciones de salud de la ciudad de Córdoba, Argentina}

A violência laboral sobre trabalhadores/as de instituições de saúde na cidade de Córdoba, Argentina

La violence au travail envers les travailleurs/euses dans les établissements de santé de Córdoba, en Argentine

Workplace violence against health care workers in Cordoba city, Argentina

Gabriel Acevedo, Alejandra Farias, Julieta Sanchez y Yanina Petiti

\section{CpenEdition}

Journals

Edición electrónica

URL: http://journals.openedition.org/laboreal/8985

DOI: $10.4000 /$ laboreal.8985

ISSN: 1646-5237

Editor

Universidade do Porto

Referencia electrónica

Gabriel Acevedo, Alejandra Farias, Julieta Sanchez y Yanina Petiti, « La violencia laboral hacia trabajadores/as de instituciones de salud de la ciudad de Córdoba, Argentina », Laboreal [En línea] Volume $6 \mathrm{~N}^{0} 1$ | 2010, Publicado el 01 julio 2010, consultado el 10 octubre 2019. URL : http:// journals.openedition.org/laboreal/8985; DOI : 10.4000/laboreal.8985

Este documento fue generado automáticamente el 10 octubre 2019.

Laboreal está licenciado com uma Licença Creative Commons - Atribuição-NãoComercial 4.0 Internacional. 


\section{La violencia laboral hacia trabajadores/as de instituciones de salud de la ciudad de Córdoba, Argentina}

A violência laboral sobre trabalhadores/as de instituições de saúde na cidade de Córdoba, Argentina

La violence au travail envers les travailleurs/euses dans les établissements de santé de Córdoba, en Argentine

Workplace violence against health care workers in Cordoba city, Argentina

Gabriel Acevedo, Alejandra Farias, Julieta Sanchez y Yanina Petiti

\section{NOTA DEL EDITOR}

Manuscrito recebido em : Março/2010

Aceite após peritagem : Junho/2010

\section{Introducción}

Se entiende por violencia "el uso deliberado de la fuerza física o el poder, ya sea en grado de amenaza o efectivo, contra uno mismo, otra persona, un grupo o comunidad, que cause o tenga muchas probabilidades de causar lesiones, muerte, daños psicológicos, trastornos del desarrollo o privaciones" (OMS, 2002). La violencia ha aumentado significativamente en los últimos decenios, afectando a toda la población ; sin respetar edad, raza, condición socioeconómica, educación, religión, género y lugar. Este señalamiento da cuenta de lo extendido del problema, no obstante cabe resaltarse 
que afecta a los distintos grupos poblacionales de un modo diferente, existiendo unos más vulnerables que otros.

El fenómeno de la violencia es complejo, por lo que resulta una tarea complicada desglosar las diferentes formas de violencia y caracterizarlas, así como establecer sus causas y consecuencias. Buinic y Cols (1999) enfatizan que existen varias formas o tipos de violencia según los aspectos que se consideran: individuos afectados; agentes de violencia; motivos; relación existente entre agresor y agredido, naturaleza de la agresión, entre otros.

3 En el lugar de trabajo, la violencia -sea física o psicológica- ha adquirido gran relevancia tanto en los países industrializados como en los países en desarrollo, alcanza diversos contextos laborales y grupos profesionales y afecta la dignidad de las personas. La violencia es una fuente de desigualdad, estigmatización y conflicto en el trabajo (Paravic, Soauzo \& Burgos, 2004). La violencia en el lugar de trabajo puede ser interna (la que tiene lugar entre los trabajadores), o externa (la que tiene lugar entre trabajadores y toda otra persona presente en el lugar de trabajo) (OIT, 2003a). Chappell y Di Martino (1998) definen a la violencia en el trabajo como : "cualquier incidente en el cual un trabajador es abusado, amenazado o atacado por un miembro del público (paciente, cliente y/o compañeros de trabajo)". Asimismo, se considera que no sólo la violencia física provoca grandes impactos en la salud del trabajador, sino aquella que se inflige a través de un comportamiento repetitivo que gradualmente daña la integridad psicológica del afectado, pudiendo llegar a tener repercusiones de mayor cuantía que la violencia física (Chappell \& Di Martino, 1998). En lo que refiere a manifestaciones de violencia en lugares de

trabajo, la variedad de comportamientos es amplia y la línea que separa a los comportamientos aceptables de los no aceptables es a veces difusa. Esto se debe a que la percepción de la violencia es diversa, dependiendo del contexto y la cultura en la que se vive, lo que implica un enorme desafío para describir y definir este fenómeno. En la década del 80, en Suecia, Heins Leymann usó el concepto "mobbing” y lo aplicó a situaciones humanas de hostilidad y de acoso psicológico repetido. En ámbitos laborales se refiere a conductas destinadas a atacar a una persona o grupo en particular, elegido como "blanco" (Leymann, 1996). Para que un acoso laboral sea considerado mobbing, debe constar de tres factores : el acoso, la repetición en el tiempo y tener el objetivo de desvincular a la persona de la organización. A partir de 1990 el concepto se popularizó y se difundió por todo el mundo, dando origen a diferentes investigaciones. El término mobbing proviene de la lengua inglesa, del verbo "to mob" (atacar, asaltar) y agrupa una diversidad de comportamientos con un fondo común: la modalidad agresiva y humillante tendiente a la eliminación de un trabajador de la institución. Las formas en las que suele presentarse son variadas y de las más sutiles : el aislamiento físico del trabajador; la marginación a través de la hostilidad y la falta de comunicación; la utilización de conceptos peyorativos; la asignación de tareas humillantes, descalificadoras o de muy difícil concreción; el menoscabo de la imagen del empleado frente a sus colegas, clientes o superiores; los cambios continuos, entre otras.

5 En Francia, Marie France Hirigoyen (2001) define el concepto "acoso moral en el trabajo" como "cualquier manifestación de una conducta abusiva (gesto, palabras, comportamiento, actitud...) que atenta por su repetición o sistematización, contra la dignidad o integridad psíquica o física de una persona, poniendo en peligro su empleo o degradando el ambiente de trabajo" También se ha considerado al "acoso laboral o 
moral" cuando el empleador o superior aísla sin motivo al trabajador de sus compañeros, le asigna tareas inútiles, lo insulta con frecuencia o lo amenaza constantemente con el despido.

6 El trabajo en los servicios de atención de la salud se distingue de otros ámbitos laborales debido a que en estos espacios se trabaja con personas. Así, la actividad que el personal de salud efectúa contiene riesgos específicos que al momento de interactuar, pueden ser generadores de accidentes o enfermedad para el conjunto de los trabajadores y para cada uno de ellos. Dentro de estos riesgos los más estudiados son los biológicos (infecciosos). Sin embargo, las situaciones que más transgreden la integridad física y mental de los trabajadores de la salud son sus condiciones de trabajo y las formas como está organizado (Yassi \& Warshaw, 1998).

7 El informe elaborado por Chappell y Di Martino en la Unión Europea, basado en 15.800 entrevistas en 15 de sus países miembros, describe que un $4 \%$ de los trabajadores fueron objeto de violencia psíquica; un $2 \%$ sufrieron acoso sexual y un $8 \%$ fueron víctimas de intimidación y hostigamiento. Un de los aspecto a destacar del estudio son los datos que relacionan la vivencia de situaciones de violencia con el género, la juventud y sectores especiales en riesgo. Sin bien no existen ocupaciones que estén inmunes a la violencia en el trabajo, esta es mayor en el sector de la salud (hospitales, clínicas y sanatorios) (Chappell \& Di Martino, 1998).

Investigaciones realizadas en los ambientes sanitarios establecen que los contextos de atención en salud no están libres del flagelo de la violencia, siendo los servicios de salud uno de los ambientes laborales más expuestos a actos agresivos de cualquier tipo (Burgos \& Paravic 2003). Se estima que cerca del $25 \%$ de los accidentes violentos en el trabajo ocurren en el sector salud y más del $50 \%$ de los trabajadores del área ya experimentó incidentes de esta naturaleza (CIE, 1999).

9 En Argentina, no se ha establecido la magnitud del problema. Se desconoce con qué frecuencia los trabajadores de los servicios de salud sufren manifestaciones de violencia en su ámbito laboral. Por ello, este trabajo tiene por objetivo estimar con qué frecuencia los trabajadores de la salud de la ciudad de Córdoba reconocen manifestaciones de acoso laboral, acoso sexual, agresión y/o discriminación, en su ámbito de trabajo.

\section{Metodología}

\subsection{Tipo de Estudio}

10 A fin de cumplir con el objetivo planteado se diseñó un estudio cuantitativo, descriptivo de corte transversal. La investigación se llevó a cabo en establecimientos de salud de la ciudad de Córdoba, Argentina.

\subsection{Población y Muestra}

11 El universo de este estudio estuvo constituido por trabajadores de la salud de la ciudad de Córdoba que están empleados en instituciones sanitarias estatales y privadas. Entre ellos se escogieron, siguiendo criterios de accesibilidad, doce establecimientos, la mitad de dependencia estatal y la otra mitad de dependencia privada. Se adoptó este mecanismo de elección de las instituciones en función de las complejidades e 
implicancias de la temática abordada. Los establecimientos elegidos, suman un total de 2140 trabajadores; de los cuales, 1220 pertenecían a instituciones de dependencia publica y 920 a instituciones de dependencia privada; de ellos se obtuvo una muestra aleatoria de 336 trabajadores.

La muestra estuvo conformada por trabajadores de ambos sexos, entre 18 y 61 años de edad, de diferentes ocupaciones/ profesiones, que accedieron voluntariamente a responder la encuesta. Al procesar los datos obtenidos se advirtió que en 15 casos los cuestionarios carecían de información en la mayoría de las variables, por lo cual se decidió no contemplarlos en el análisis final.

\subsection{Instrumento de recolección de datos}

13 Se administró un instrumento diseñado y validado por investigadores de la Escuela de Salud Pública de la Universidad de Chile, (Escuela de Salud Pública, Universidad de Chile. 2008. compuesto por preguntas de respuestas cerradas. Se realizó una prueba piloto en 40 trabajadores, no encontrándose dificultades en su aplicación ni sugerencias para su modificación. El instrumento se divide en 4 bloques: 1. Datos sociodemográficos ; 2 . Acoso laboral en el trabajo ; 3 . Acoso sexual en el trabajo y 4. Agresiones físicas y discriminación. Con el propósito de orientar al encuestado los bloques 2, 3 y 4 estuvieron encabezados con definiciones de los términos correspondientes. La encuesta fue autoadministrada, confidencial y anónima, aplicada durante el período comprendido entre los meses de junio a septiembre del año 2009.

\subsection{Variables Estudiadas}

14 Acoso laboral toda conducta abusiva (gesto, palabras, comportamiento, actitud...) que atenta por su repetición o sistematización, contra la dignidad o integridad psíquica o física de un trabajador, poniendo en peligro su empleo o degradando el ambiente de trabajo (Hirigoyen, 2001).

Acoso sexual El "acoso sexual" es una insinuación o exigencia sexual que, al ser rechazado por quien la recibe, pone en riesgo su estabilidad laboral u oportunidades de trabajo (OIT, 2003b). Agresiones físicas El uso deliberado de la fuerza física contra una persona, que cause o tenga muchas probabilidades de causar lesiones (OMS, 1996).

Agresiones psicológicas Ocurre cuando un individuo es víctima de insultos, amenazas, gritos, o cualquier conducta abusiva (gesto, palabras, comportamiento, actitud...) que atenta por su repetición o sistematización, contra la dignidad o integridad psíquica, poniendo en peligro su empleo o degradando el ambiente de trabajo (OMS, 1996).

Discriminación es el acto de hacer una distinción o segregación que atenta contra la igualdad de oportunidades. Se utiliza para referirse a la violación de la igualdad de derechos para los individuos por cuestiones sociales, raciales, religiosas, orientaciones sexuales, género, entre otras (OMS, 1996).

\subsection{Análisis de la información}

18 El análisis de los datos se realizó mediante el paquete estadístico Statistical Package for the Social Sciences (SPSS) versión 15 para Windows. Se realizaron análisis estadístico descriptivo y de asociaciones de variables. En primer lugar, se efectuó un análisis 
descriptivo de los datos con el objeto de explorar el comportamiento de las variables en general (frecuencias y medidas de tendencia central). Para este análisis se obtuvieron los porcentajes en cada una de las categorías de las variables. Posteriormente se realizó el cruzamiento de variables. Con esta fialidad, se elaboraron tablas de contingencia utilizando el estadístico chicuadrado. El punto crítico de rechazo de la hipótesis nula fue el valor alfa $=0.05$.

\section{Resultados}

\subsection{Características sociodemográficas de la muestra}

Como puede observarse en la tabla 1, la muestra de trabajadores/as estudiados quedó conformada, en cuanto a la distribución por sexo, en un $68,8 \%$ por mujeres; Concordando esto con la composición estimada por Pautassi y Burijovich (2005), donde, el $70 \%$ de trabajadores del sector salud de Córdoba, eran mujeres.

Tabla 1 : Características sociodemográficas de los trabajadores encuestados.Fuente : Investigación La violencia laboral hacia trabajadores/as de instituciones de salud de la ciudad de Córdoba, Argentina.

\begin{tabular}{|c|c|c|c|c|c|c|c|}
\hline & & \multicolumn{2}{|c|}{$\begin{array}{l}\text { Instituciones } \\
\text { Estatales }\end{array}$} & \multicolumn{2}{|c|}{$\begin{array}{l}\text { Instituciones } \\
\text { Privadas }\end{array}$} & \multicolumn{2}{|c|}{ Total } \\
\hline & & $f$ & $\%$ & f & $\%$ & f & $\%$ \\
\hline \multirow{2}{*}{ ๙ื่ } & Femenino & 131 & 40,8 & 90 & 28 & 221 & 68,8 \\
\hline & Masculino & 52 & 16,2 & 48 & 15 & 100 & 31,2 \\
\hline \multirow{2}{*}{ 晃 } & 17 a 35 años & 98 & 30,5 & 104 & 32,4 & 202 & 62,9 \\
\hline & 36 a 61 años & 85 & 26,5 & 34 & 10,6 & 119 & 37,1 \\
\hline \multirow{4}{*}{$\begin{array}{l}\text { 은 } \\
\frac{\text { gू }}{3} \\
\text { o }\end{array}$} & Médicos/as & 43 & 13,4 & 26 & 8,1 & 69 & 21,5 \\
\hline & Enfermeras/os & 47 & 14,6 & 49 & 15,3 & 96 & 29,9 \\
\hline & Residentes: & 41 & 12,8 & 36 & 11,2 & 77 & 24 \\
\hline & $\begin{array}{c}\text { Otros } \\
\text { trabajadores }\end{array}$ & 52 & 16,2 & 27 & 8,4 & 79 & 24,6 \\
\hline
\end{tabular}

En lo que refiere a su distribución etárea, el 62,9\% de los participantes tenía entre 18 y 35 años de edad y el resto entre 36 y 61 años. La distribución por ocupación/profesión muestra que el $29,9 \%$ fueron enfermeras/os ; el $24,0 \%$ residentes ; el 21,5\% médicos/as y el $24,6 \%$ corresponden a otras ocupaciones y profesiones de la salud. Esta relación médico/as - enfermera/ os (integrando en la categoría medico/as a estos más los residentes) fue semejante a la media nacional, que se sitúa en dos médico/as por cada enfermera/os, relación esta que se encuentra invertida cuando se la compara con la 
existente en otros países de América Latina. El 57,0 \% de los participantes trabajaba en instituciones de dependencia estatal.

\subsection{Tipos de violencia suferidas en el trabajo}

21 Se preguntó a los encuestados si habían sufrido manifestaciones de violencia en su actual lugar de trabajo en los últimos 12 meses. En el grafico 1 puede observarse las frecuencias con que los trabajadores refirieron haber padecido agresiones psicológicas, agresiones físicas, acoso laboral y sexual. Las agresiones de tipo psicológica resultan las más frecuentes, seguidas por las de acoso laboral, es decir las de tipo psicológico se presentaron con mayor frecuencia que las físicas.san sufrir más casos de acoso laboral en su lugar de trabajo que aquellos trabajadores que lo hacen en instituciones privadas, con diferencias estadísticamente significativas $(p<0,05)$.

Grafico 1 : Tipos de Violencia Laboral sufridas por Trabajadores de Salud. Fuente : Investigación La violencia laboral hacia trabajadores/as de instituciones de salud de la ciudad de Córdoba, Argentina.

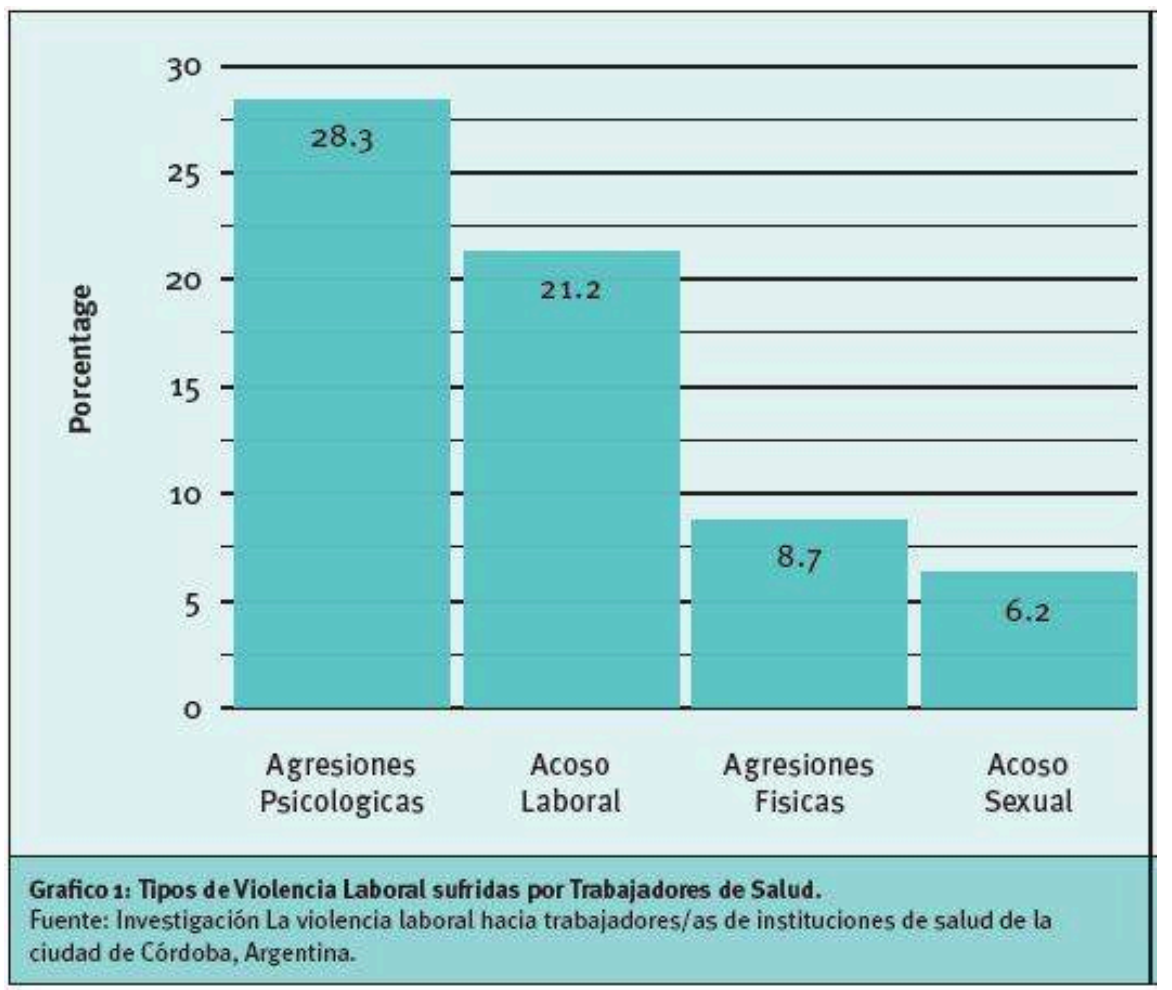

\subsection{Manifestaciones de Violencia según atributos del/la trabajador/}

a

Las manifestaciones de violencia fueron estudiadas según atributos de los/las trabajadores/as tales como Sexo, Edad, Ocupación y tipo de establecimiento en el que se desempeñan, cuya distribución de frecuencias puede observarse en la tabla 2. Las mujeres refieren haber padecido las diversas formas de violencia estudiadas en mayor proporción que los varones. Los/as trabajadores/as más jóvenes han sufrido (con excepción del acoso laboral) más frecuentemente la violencia que lo mayores. La 
ocupación en enfermería es en general la que más se ve afectada. Quienes trabajan en establecimientos públicos sufren más frecuentemente la violencia que los que lo hacen en instituciones privadas

Tabla 2 : Distribución de respuestas sobre manifestaciones de violencia laboral según frecuencias por Edad, Ocupación y tipo de establecimiento. Fuente : Investigación La violencia laboral hacia trabajadores/as de instituciones de salud de la ciudad de Córdoba, Argentina.

\begin{tabular}{|c|c|c|c|c|c|}
\hline & & 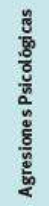 & 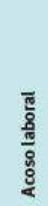 & 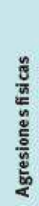 & 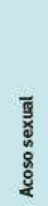 \\
\hline & & $\%$ & $\%$ & $\%$ & $\%$ \\
\hline \multirow{2}{*}{ ڤ్ } & Mujeres & 18,6 & 18,1 & 5,6 & 4,3 \\
\hline & Varones & 9,7 & 3,1 & 3,1 & 1,9 \\
\hline \multirow{2}{*}{ 迈 } & 18 a 35 años & 17,7 & 13,1 & 4 & 3.4 \\
\hline & 36 a 61 años & 10,6 & 8,1 & 4,7 & 2,8 \\
\hline \multirow{4}{*}{$\frac{.5}{\frac{\delta}{3}}$} & Médicos & 6,8 & 3,8 & 2,8 & 0,9 \\
\hline & Enfermeras & 7,8 & 9 & 2,2 & 1,9 \\
\hline & Residentes & 7,8 & 4,6 & 2,1 & 1,6 \\
\hline & Otros trabajadores de la salud & 5,9 & 3,8 & 1,6 & 1,8 \\
\hline \multirow{2}{*}{$\stackrel{8}{\frac{\delta}{2}}$} & Pública & 18,4 & 12,2 & 6,5 & 3,1 \\
\hline & Privada & 9,9 & 9 & 2,2 & 3,1 \\
\hline \multicolumn{6}{|c|}{$\begin{array}{l}\text { Tabla 2: Distribución de respuestas sobre manifestaciones de violencia laboral según frecuencias } \\
\text { por Edad, Ocupación y tipo de establecimiento. } \\
\text { Fuente: Investigación La violencia laboral hacia trabajadores/as de instituciones de salud de la ciudac } \\
\text { de Córdoba, Argentina. }\end{array}$} \\
\hline
\end{tabular}

Se hallaron diferencias estadísticamente significativas $(\mathrm{p}<0,05)$ en relación a haber sufrido acoso sexual según el sexo del trabajador/a, por lo que puede inferirse que las mujeres han vivenciado más casos de acoso sexual en su lugar de trabajo que los varones. También se hallaron diferencias estadísticamente significativas $(p<0,05)$ según la edad del encuestado, los más jóvenes (trabajadores cuya edad está comprendida entre 18 y 35 años) manifiestan haber sido acosados laboralmente, insultados o agredidos físicamente en su lugar de trabajo, durante el último año más frecuentemente que los trabajadores cuya edad está comprendida entre 36 y 61 años. Los trabajadores que se desempeñan en instituciones públicas, expresan sufrir más casos de acoso laboral en su lugar de trabajo que aquellos trabajadores que lo hacen en instituciones privadas, con diferencias estadísticamente significativas $(\mathrm{p}<0,05)$

\subsection{Reconocimiento de manifestaciones de violencia en el ámbito laboral}

Se interrogó a los trabajadores estudiados si conocían sobre la ocurrencia de hechos de violencia en su ámbito de trabajo, durante el último año, independientemente de que ellos los hubieran padecido. Las agresiones verbales, el acoso laboral y las manifestaciones de discriminación constituyen expresiones de violencia en el trabajo que son reconocidas con una elevada frecuencia por parte de los trabajadores de salud. 

encuestados y el tipo de institución con la pregunta ¿Han ocurrido casos de acoso sexual en su trabajo, en los últimos 12 meses? Así, las mujeres y los trabajadores de instituciones públicas percibieron la existencia de acoso sexual más frecuentemente que los trabajadores de instituciones privadas y los varones.

Grafico 2 : Grado de reconocimiento de manifestaciones de violencia laboral. Fuente : Investigación La violencia laboral hacia trabajadores/as de instituciones de salud de la ciudad de Córdoba, Argentina.

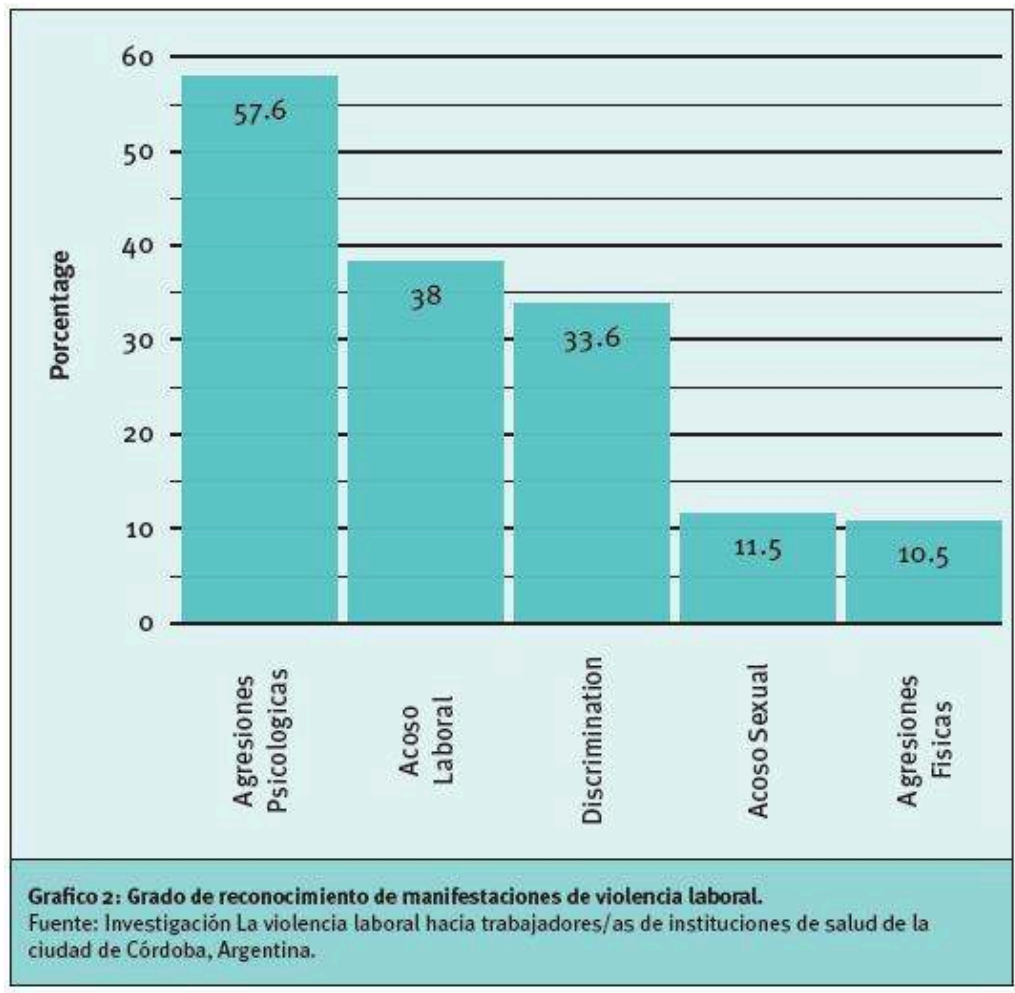

\subsection{Respuesta institucional}

A los encuestados que respondieron que en su lugar de trabajo habían ocurrido casos de acoso laboral y acoso sexual se les indagó si conocían que se hubiera realizado una investigación de la situación. De los que respondieron que en su lugar de trabajo habían ocurrido casos de acoso laboral el 85,2\% respondió que no se hizo una investigación posterior de la situación, en tanto que el $78,4 \%$ de los que refirieron que habían ocurrido casos de acoso sexual señaló que no se investigó la situación. (Tabla 3). A los encuestados que no sabían de situaciones de acoso sexual en su trabajo en los últimos 12 meses, se preguntó si se sentían protegidos por la institución frente a estas situaciones. El $61,1 \%$ respondió negativamente. Se encontraron diferencias 
estadísticamente significativas $(\mathrm{p}<0,05)$ en relación al tipo de institución y la realización de una investigación de los hechos de violencia, siendo que en las estatales se investigan más este tipo de situaciones.

Tabla 3. Percepción de la respuesta Institucional según tipo de Violencia. Fuente : Investigación La violencia laboral hacia trabajadores/as de instituciones de salud de la ciudad de Córdoba, Argentina

\begin{tabular}{|c|c|c|c|c|}
\hline \multirow{3}{*}{ Tipo de Violencia } & \multicolumn{4}{|c|}{ Medidas antropométricas } \\
\hline & \multicolumn{2}{|c|}{ Se Investigó } & \multicolumn{2}{|c|}{ No se Investigó } \\
\hline & Estatal & Privada & Estatal & Privada \\
\hline Acoso Laboral & $4,7 \%$ & $3.7 \%$ & $52,3 \%$ & $39,3 \%$ \\
\hline Acoso Sexual & $2,8 \%$ & $2,5 \%$ & $54,2 \%$ & $40,5 \%$ \\
\hline \multicolumn{5}{|c|}{$\begin{array}{l}\text { Tabla 3. Percepción de la respuesta Institucional según tipo de Violencia. } \\
\text { Fuente: Investigación La violencia laboral hacia trabajadores/as de instituciones de salud de la ciuda } \\
\text { de Córdoba, Argentina. }\end{array}$} \\
\hline
\end{tabular}

\section{Discusión}

Las diversas manifestaciones de violencia en el trabajo, estudiadas en esta investigación, afectan a los trabajadores que se desempeñan en establecimientos de salud de dependencia estatal y privada de la ciudad de Córdoba.

Los insultos y otras formas de agresión verbal fueron el tipo de violencia identificada con mayor frecuencia. Los casos donde los trabajadores han sido insultados o agredidos verbalmente $(28,3 \%)$, son de menor frecuencia a los encontrados en una investigación realizada en trabajadores de la salud de Jamaica donde observaron que en términos de violencia psicológica, el abuso verbal presenta una frecuencia del 38,6\%, en tanto que son similares las frecuencias en lo que refiere a agresiones físicas, ya que en nuestro estudio se estimó en un 8,7 \% y en el estudio jamaiquino en un 8 \% (Jackson \& Asheley, 2005).

En relación al acoso laboral, más de un $20 \%$ de los trabajadores expresaron haberlo sufrido en alguna oportunidad. Esta frecuencia es mayor a la encontrada en investigaciones realizadas en Río de Janeiro, Brasil, donde se observó que el 16,8\% de los trabajadores de la salud encuestados han sido víctima de acoso laboral (Hungria, Veiga \& Peixoto, 2008). Si bien en esa investigación se aclara que su muestra fue tomada en el año 2001 y analizada en el 2008, lo que podría explicar una menor percepción, ya que esta problemática ha comenzado a incrementar su visibilidad en los últimos años. No obstante, en un estudio realizado por Paravic, Valenzuela y Burgos (2003), en Chile, hallaron que la violencia percibida por trabajadores de atención primaria supera el $50 \%$, de modo que pareciera ser este un fenómeno que adquiere gran envergadura en el sector salud.

31 La magnitud de los casos de acoso sexual que han sufrido los trabajadores de salud $(6,2 \%)$ de Córdoba estudiados en este trabajo, es similar al encontrado en los 
trabajadores de la salud en Chile, cuya proporción se situó en el $7 \%$ (Paravic, Valenzuela \& Burgos, 2003), aunque muy inferior al 37,1\% encontrado en un estudio realizado en enfermeras que trabajan en ocho hospitales del Ministerio de Salud de Turquía (Celik, 2007).

Las mujeres declaran en un mayor porcentaje haber vivido experiencias de acoso laboral, acoso sexual, insultos o agresiones físicas y discriminación respecto de los hombres, en sus actuales lugares de trabajo. Otro aspecto que cabe destacarse es que los trabajadores más jóvenes percibieron la existencia de acoso laboral más frecuentemente que los trabajadores cuya edad está comprendida entre 36 y 61 años, situación que podría relacionarse con la ubicación que tienen en la estructura jerárquica de la institución, en particular de la hospitalaria, y que los expone en mayor medida a situaciones de violencia generada por profesionales de mayor jerarquía, o bien porque entre los trabajadores de mayor antigüedad se va produciendo un fenómeno de acostumbramiento a las situaciones de acoso laboral y por ello las perciben con menor frecuencia (Hijar, López \& Blanco, 1997).

Además, se pudo establecer que los trabajadores que se desempeñan en instituciones de dependencia estatal manifestaron conocer y sufrir más casos de acoso laboral en su lugar de trabajo que aquellos trabajadores que lo hacen en instituciones privadas. Podría esto estar vinculado a diversos condicionantes, tales como el mayor grado de conflictividad laboral que tienen los establecimientos estatales, a que asisten a una población con mayores carencias, a una mayor restricción de recursos e insumos que resienten la atención, entre otros. Cabe aclarar que estos factores condicionantes de la violencia podrían explicar las diferencias de percepciones encontradas. Cabe destacar que lo antes mencionado son solo hipótesis, ya que no han sido estudiadas en la presente investigación.

El grado de reconocimiento por parte de los trabajadores de la ocurrencia en su lugar de trabajo de situaciones de violencia fue elevado. El 57,6 \% declara conocer situaciones de insultos y otras agresiones verbales y el $38 \%$ casos de acoso laboral que afectaron a quienes se desempeñaban en su ámbito de trabajo. Si bien estas frecuencias son menores a las reportadas por Lanza ML y col que encontraron que en centros de atención de la salud el 72,8 \% de los trabajadores informaron de al menos un incidente de violencia no física y el 21,3\% informaron de al menos un incidente de violencia física (Lanza, Zeiss \& Rierdan, 2006).

\section{Conclusiones}

Con base en la información obtenida sobre la ocurrencia de las diversas manifestaciones de violencia en el trabajo puede señalarse que el acoso laboral, el acoso sexual, las agresiones psicológicas y físicas están frecuentemente presentes en instituciones del sector salud en la ciudad de Córdoba, afectando a un número significativo de trabajadores y constituyen riesgos ocupacionales de gran relevancia para quienes allí se desempeñan.

Las situaciones de violencia encontradas en la presente investigación son principalmente del tipo psicológico, como las agresiones verbales y el acoso laboral, que afectan predominantemente a las mujeres y a los más jóvenes, siendo percibidas con mayor frecuencia por quienes trabajan en establecimientos estatales. Las 
manifestaciones de violencia física y sexual, también están presentes, aunque con una frecuencia más baja que las psicológicas.

La respuesta institucional ante este fenómeno puede valorarse como muy insuficiente, especialmente en los establecimientos de dependencia privada, dado que en la mayoría de los casos no se realizaron investigaciones de los hechos de violencia ocurridos. Posiblemente por ello, los/as trabajadores/as expresan no sentirse protegidos por la institución en la que trabajan.

Así, los hallazgos de este trabajo permiten estimar la magnitud de los distintos tipos de violencia ejercida hacia los trabajadores y trabajadoras de salud, generando las bases para futuras investigaciones que se orienten a identificar que grupos de trabajadores presentan una mayor vulnerabilidad a sufrir actos de violencia, cuáles son los factores condicionantes, los mecanismos de producción y las consecuencias más directas de la misma, entendiendo que la obtención de un mayor reconocimiento de la magnitud y los alcances de la violencia en el trabajo del sector sanitario, sus condicionantes e implicancias posibilitarán el diseño de estrategias, que contemplando las particularidades y especificidades del sector en nuestro medio, propicien ambientes de trabajo más saludables.

\section{BIBLIOGRAFÍA}

Buinic, M., Morrison, A. \& Shifter, M. (1999). La violencia en América Latina y el Caribe : un Marco de Referencia para la Acción. Washington D.C. : Banco Interamericano de Desarrollo.

Burgos, M. \& Paravic, T. (2003). Percepción de violencia de los pacientes hospitalizados en los servicios de medicina y cirugía de un hospital público. Ciencia y enfermería, 9(2), 29-42.

Celik, Y. \& Celik, SS. (2007). Sexual harassment against nurses in Turkey. J Nurs Scholars ; 39(2), 200-6.

Consejo Internacional de Enfermería. (1999). El incremento de la violencia en el lugar de trabajo es una amenaza para la enfermería y la prestación de los cuidados de salud (Comunicado de prensa). Consultado en Julio, 23, 2007, de http://www.icn.ch/prviolence_99sp.htm Chappell, D. \& Di Martino, V. (1998). Violence at work. Ginebra : Oficina Internacional del Trabajo. Escuela de Salud Pública, Facultad de Medicina, Universidad de Chile. (2008). Validación y estandarización de encuesta psicosocial en la población chilena, ISTAS 21. Superintendencia de Seguridad Social de Chile.

Hijar, M., López, M.V. \& Blanco, J. (1997). La violencia y sus repercusiones en la salud ; reflexiones teóricas y magnitud del problema en México. Salud Pública de México. 39(6), 1-8.

Hirigoyen, MF. (2001). L'assetjament moral a la feina. Distingir el cert del fals. Ed. 62. Barcelona : Paidós. 
Hungria Xavier, A. C., Veiga Barcelos, C.R., Peixoto Lopes, J. et al. (2008). Assédio moral no trabalho no setor saúde no Rio de Janeiro : algumas características. Revista Brasileira de Saúde Ocupacional, São Paulo, 33 (117), 15-22.

Jackson, M., Ashley, D. (2005). Physical and psychological violence in Jamaica's health sector. Revista Panameña de Salud Pública. 18 (2), 114-21.

Lanza, M., Zeiss R.A. \& Rierdan, J. (2006). Nonphysical violence : a risk factor for physical violence in health care settings. A AOHN J ;4(9), 397-402.

Leymann, H. (1996). Mobbing. The persecution au travail. Paris : Ed. Du Seuil.

Organización Internacional del Trabajo, OIT/ICN/OMS/ISP. (2003a). Relationship between work stress and workplace violence in the health sector. Ginebra : Documento de trabajo del Programa conjunto de la OIT/ICN/OMS/ISP sobre la violencia en los lugares de trabajo del sector de salud.

Organización Internacional del Trabajo. (2003b). Guía sindical para abordar el acoso sexual en el trabajo. Madrid : Secretaría Confederal de la Mujer de CC.OO. Consultado en Agosto, 26 2008, de http://www.ccoo.es/csccoo/menu.do?Informaci\%C3\%B3n\%3APublicaciones

Organización Mundial de la Salud. (1996). Global Consultation on Violence and Health. Violence : a public health priority. Ginebra : Documento WHO/EHA/SPI.POA.2.

SPSS para Windows. Versión 15.0.0. (2006). Chicago : SPSS Inc. (programa informático en CD-ROM). Disponible en SPSS Inc. Página web de SPSS disponible en : http://www.spss.com

Paravic, T., Valenzuela, S., Burgos, M. (2004). Violencia percibida por trabajadores de atención primaria de la salud. Ciencia y enfermería, 10 (2), 53-65.

Pautassi, L. \& Burijovich, J. (2005). Calidad de empleo y calidad de la atención en salud en Córdoba, Argentina. Aportes para políticas laborales más equitativas. Chile : CEPAL, Naciones Unidas.

Yassi, A., Warshaw, L.J. (1998). Asistencia Sanitaria : Naturaleza y Problemas de Salud en el Trabajo. In : Enciclopedia de Salud y Seguridad en el Trabajo. $4^{a}$ ed. Ginebra : Organización Internacional del Trabajo.

\section{RESÚMENES}

La violencia laboral se manifiesta de diversas formas : acoso laboral, acoso sexual, agresión física y psicológica y discriminación. Diversas investigaciones advierten que los trabajadores de salud son víctimas del $25 \%$ de los eventos de violencia laboral registrados. En Argentina la magnitud del problema no es bien conocida, por lo que el objetivo de este trabajo fue estimar la frecuencia de la violencia hacia los trabajadores de la salud. El estudio realizado es descriptivo de corte transversal. La muestra se seleccionó aleatoriamente; se administró una encuesta a 336 trabajadores de establecimientos de salud de Córdoba. El 21,2 \% de los encuestados refirieron haber padecido acoso laboral, el 6,2\% acoso sexual, agresiones físicas el 8,7\% y el 28,3\% agresiones psicológicas. Se concluye que distintos tipos de violencia están frecuentemente presentes en las instituciones estudiadas; los hechos de violencia son escasamente investigados y que los trabajadores refieren no sentirse protegidos por la institución.

A violência laboral manifesta-se de diversas formas: assédio laboral, assédio sexual, discriminação, agressões. Investigações alertam para o facto de que os trabalhadores da saúde são vítimas de 25 \% dos eventos de violência laboral registados. Porém, na Argentina, a magnitude do problema não é bem conhecida. O objectivo deste trabalho foi o de fazer uma es- timativa da frequência de violência infligida aos trabalhadores de saúde. O estudo realizado é descritivo 
transversal. A amostra foi seleccionada de forma aleatória. Administrou-se um inquérito a 336 trabalhadores de estabelecimentos de saúde em Córdoba. 21,2 \% dos respondentes referiram ter sofrido assédio laboral, $6.2 \%$ referiram assédio sexual, 8,7 \% agressões físicas e 28,3\% agressões psicológicas. Conclui-se que a violência está presente sob diversas formas nas instituições estudadas. Na maioria dos casos estas situações não foram investigadas e os trabalhadores expressam não sentir a protecção da instituição.

La violence au travail se présente sous différentes formes : le harcèlement moral, le harcèlement sexuel, la discrimination et les agressions. Les enquêtes montrent que les professionnels de la santé correspondent à $25 \%$ des victimes enregistrées suite à des faits de violence au travail. En Argentine, l'ampleur du problème est mal connue, et l'objectif de cette étude a été de mieux la circonscrire. L'étude est descriptive et transversale. L'échantillon a été choisi au hasard, un sondage a été administré à 336 travailleurs dans des établissements de santé de Cordoba. 21,2\% des répondants ont déclaré avoir subi des brimades, $6,2 \%$ un harcèlement sexuel, 8,7\% des violences physiques, et $28,3 \%$ des agressions psychologiques. Nous concluons que la violence est présente de différentes manières dans les institutions analysées. Dans la plupart des cas, il n'y a pas eu d'enquête concernant les faits et les travailleurs disent qu'ils ne se sentent pas protégés par l'institution.

Workplace violence occurs in different types: mobbing, sexual harassment, physical and psychological aggression and discrimination. Research shows that health workers are victims of 25 per cent of the reported cases of workplace violence. In Argentina, the dimension of the problem is little known; therefore, the objective of the present study is to determine the frequency of violence against health workers. A crosssectional study was designed and a random sample selected ; a survey was administered to 336 workers in health facilities in Cordoba. 21.2 per cent of respondents reported having experienced mobbing; 6.2 per cent experienced sexual harassment, while 8.7 per cent and 28.3 per cent suffered physical violence and psychological aggression, respectively. The following conclusions were reached : different types of violence are often present in the centers studied, little investigation has been carried out and the health workers responded that they feel unprotected.

\section{ÍNDICE}

Keywords: workplace violence, sexual harassment, mobbing, health workers

Palavras-chave: violência laboral, assédio sexual, assédio laboral, trabalhadores da saúde

Palabras claves: violencia laboral, acoso sexual, acoso laboral, trabajadores de la salud

Mots-clés: violence au travail, harcèlement au travail, harcèlement sexuel, professionnels de la santé

\section{AUTORES}

\section{GABRIEL ACEVEDO}

Escuela de Salud Pública, Facultad de Ciencias Médicas, Universidad Nacional de Córdoba. Enrique Barros esq. Enfermera Gordillo Gómez. Ciudad Universitaria, 5000, Córdoba - Argentina geacevedo_esp@fcm.unc.edu.ar 
ALEJANDRA FARIAS

Escuela de Salud Pública, Facultad de Ciencias Médicas, Universidad Nacional de Córdoba.

Enrique Barros esq. Enfermera Gordillo Gómez. Ciudad Universitaria, 5000, Córdoba - Argentina afarias@fcm.unc.edu.ar

\section{JULIETA SANCHEZ}

Escuela de Salud Pública, Facultad de Ciencias Médicas, Universidad Nacional de Córdoba.

Enrique Barros esq. Enfermera Gordillo Gómez. Ciudad Universitaria, 5000, Córdoba - Argentina jsanchez@fcm.unc.edu.ar

\section{YANINA PETITI}

Escuela de Salud Pública, Facultad de Ciencias Médicas, Universidad Nacional de Córdoba.

Enrique Barros esq. Enfermera Gordillo Gómez. Ciudad Universitaria, 5000, Córdoba - Argentina yaninapetiti@yahoo.com.ar 